\title{
Pain and disability reported in the year following a distal radius fracture: A cohort study
} Joy C MacDermid*1,2, James H Roth ${ }^{1,3}$ and Robert S Richards ${ }^{1,3}$

Address: ${ }^{1}$ Hand and Upper Limb Centre, St. Joseph's Health Centre, London, N6A 4L6 Ontario, Canada, ${ }^{2}$ School of Rehabilitation Sciences McMaster University, Hamilton, L8S 1C7, Ontario, Canada and ${ }^{3}$ Department of Surgery, University of Western Ontario, London, Ontario, Canada

Email: Joy C MacDermid* - macderj@mcmaster.ca; James H Roth - james.roth@sjhc.london.on.ca; Robert S Richards - rrichard@uwo.ca * Corresponding author

Published: 31 October 2003

BMC Musculoskeletal Disorders 2003, 4:24
Received: 04 June 2003

Accepted: 31 October 2003

This article is available from: http://www.biomedcentral.com/I47I-2474/4/24

(C) 2003 MacDermid et al; licensee BioMed Central Ltd. This is an Open Access article: verbatim copying and redistribution of this article are permitted in all media for any purpose, provided this notice is preserved along with the article's original URL.

\begin{abstract}
Background: Distal radius fractures are a common injury that cause pain and disability. The purpose of this study was to describe the pain and disabilities experienced by patients with a distal radius fracture in the first year following fracture.
\end{abstract}

Methods: A prospective cohort study of 129 patients with a fracture of the distal radius was conducted. Patients completed a Patient-rated Wrist Evaluation at their baseline clinic visit and at 2, 3, 6 and 12 months following their fracture. The frequency/severity of pain and disabilities reported was described at each time point.

Results: The majority of patients experienced mild pain at rest and (very) severe high levels of pain with movement during the first two-months following distal radius fracture. This time is also associated with (very) severe difficulty in performing specific functional activities and moderate to severe difficulty in four domains of usual activity. The majority of recovery occurred within sixmonths, but symptoms persisted for a small minority of patients at one-year following fracture. Patients had the most difficulty with carrying ten pounds and pushing up from a chair. Resumption of usual personal care and household work preceded, and was more complete, than work and recreational participation.

Conclusions: This study demonstrated that the normal course of recovery following a distal radius fracture is one where severe symptoms subside within the first two-months and the majority of patients can be expected to have minimal pain and disability by six-months following fracture. This information can be used when planning interventions and assessing whether the progress of a patient is typical of other patients.

\section{Background}

Distal radius fractures are common injuries that cause pain and disability. Despite this, few studies have described the disability experience of patients with this type of injury. A large number of studies, most frequently case series, have reported the impairments in specific clinical parameters resulting from a distal radius fracture.
Usually these studies were conducted to describe the outcomes of a specific treatment intervention. Range of motion and grip strength scores are the most commonly reported impairments. A minority of studies on distal radius fracture has incorporated longitudinal evaluations allowing them to describe how these impairments changed over time. Even fewer studies have focused on the 
symptomatic and functional disability experience of patients. However, from studies describing the relationships between impairment and disability in this patient group, we know that the relationship is moderate at best $[1,2]$. Thus, even well-designed longitudinal studies focusing on impairment have provided little understanding of the disability experienced by patients following a distal radius fracture.

Only recently, have standardized measures indicating disability from the perspective of the patient with a wrist injury been included in reports of outcomes after distal radius fracture [3-7]. We have previously reported aggregate scores for a number of outcomes, including patient self-report scales (Patient-rated Wrist Evaluation, DASH and the SF-36) in a cohort of patients with distal radius fractures followed at specific intervals for one year [3]. This summary information can be used to compare outcome at the group or aggregate level. However, this data does not illustrate what specific aspects of function were most affected, nor what percentage of patients experienced particular difficulties.

A recent qualitative study investigated the disability experience of a selected group of wrist-injured patients [8]. A semi-structured interview process was used to obtain information about the patient's status prior to injury, specific activities which were difficult to perform, compensatory mechanisms that patients used to deal with their limited function, and the patient's self-reported main problem. These patients had symptoms which had been present for 1-164 months (average 19). A variety of activities were reported to be difficult, with the two most common reported, eating with utensils and work activities involving grasping/lifting. The most common categories reported to present difficulty to patients were: work activities (65 percent) or domestic duties (54 percent). The authors also described compensatory mechanisms used by patients to assist them in dealing with their disability, as well as a number of positive and negative effects of their wrist problem. This study provided some useful information on the types of activities that are difficult for patients following a wrist injury. However, it did not quantify the relative amount of difficulty, it did not address how it changed over time and was not specific to any given wrist pathology.

Standardized self-report forms that are specific to wristrated pain and disability, make it possible to quantify the difficulty patients are having with specific activities. The Patient-rated Wrist Evaluation (PRWE) $[9,10]$ was designed for this purpose (Figure 1). While these standardized scales do not reflect every functional activity of importance to a given individual patient, they do provide one method of gaining understanding about the experi- ence of patients with respect to specific activities or domains of life. That is, by examining the responses to individual items on standardized self-report forms, we are able to gain an understanding of the disabilities caused by a particular condition and the natural history of recovery.

Therefore, the purpose of this study was to examine the pain and disability experience of patients following a distal radius fracture and how that experience changes over time.

\section{Methods \\ Patients}

A cohort of 129 successive patients with a distal radius fracture who completed a baseline PRWE when attending hand clinic were entered into this study. Patients were excluded if they were unable to complete the PRWE due to mental incompetence or language barriers that could not be addressed using hospital or family translators (Available Cohort $=137$; Excluded because baseline form not present $=8$ ). The patients were treated by 7 different hand surgeons according to best treatment practices of a specialized hand unit. The basic principles of fracture management of the Centre include initial reduction in emergency for displaced fractures, followed by re-examination in hand clinic. A treatment plan which provided for reduction and fixation of the fracture was determined by individual physicians in consultation with patients and included a variety of options (see Table 1). An on-site hand therapy unit was available and patients were seen by therapists with treatment ranging from home programs to intensive therapy depending on the patient's needs. Patients from distant locations attended therapy in community hospitals with supportive consultations provided by hand therapists, if required. Extra-articular, partialarticular and complete articular fractures were present. See Table 1 for descriptive information on patient and treatment characteristics.

\section{Evaluations}

Patients were evaluated at their baseline hand clinic visits (following their emergency visits and initial treatment) and again at 2, 3, 6 and 12 months following their fracture. At each visit the patients completed a PRWE which was checked to ensure that all items were completed.

The PRWE has been previously described elsewhere [911,7 land is appended in Figure 1. It has been shown to be reliable, valid and responsive in patients with distal radius fractures. It has also been used to assess patients with a variety of wrist disorders [1-6].

The PRWE contains 15 items: a 5-item Pain subscale (4 questions on pain intensity and one on frequency), a 6item Specific Activities subscale and a 4-item Usual 
Name:

Date:

\section{PATIENT RATED WRIST EVALUATION}

The questions below will help us understand how much difficulty you have had with your wrist in the past week. You will be describing your average wrist symptoms over the past week on a scale of 0-10. Please provide an answer for ALL questions. If you did not perform an activity, please ESTIMATE the pain or difficulty you would expect. If you have never performed the activity, you may leave it blank.

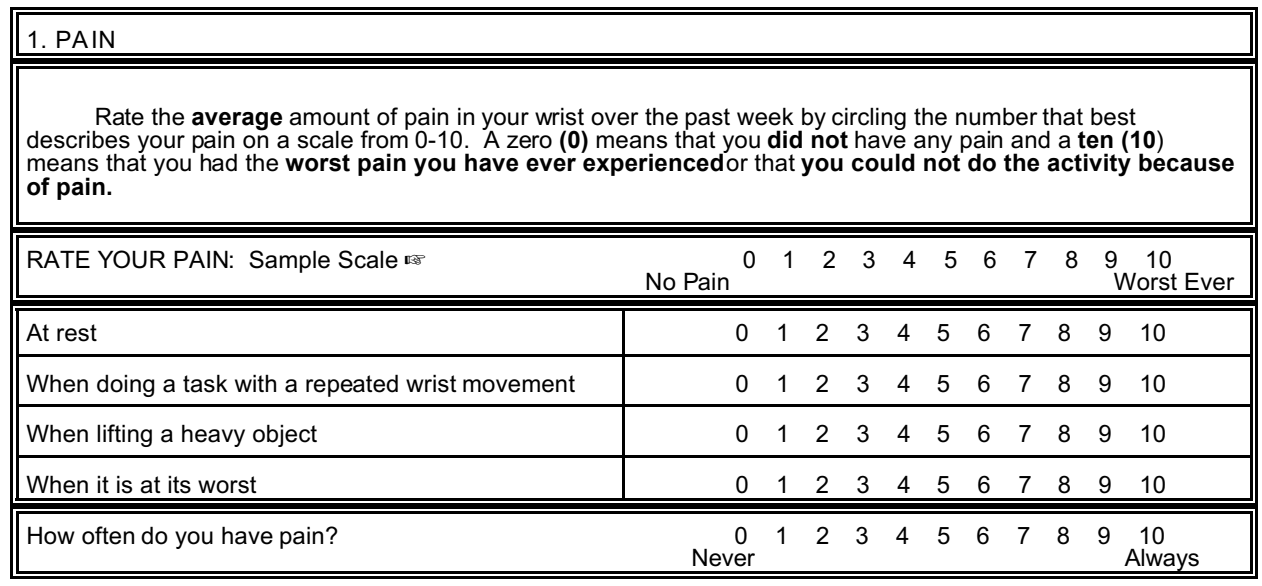

\section{FUNCTION}

\section{A. SPECIFIC ACTIVITIES}

Rate the amount of difficulty you experienced performing each of the items listed below - over the past week, by circling the number that describes your difficulty on a scale of 0-10. A zero (0) means you did not experience any difficulty and a ten (10) means it was so difficult you were unable to do it at all

Sample scale $\rightarrow \quad \begin{array}{rlllllllllll}0 & 1 & 2 & 3 & 4 & 5 & 6 & 7 & 8 & 9 & 10\end{array}$ No Difficulty $\quad$ Unable

\begin{tabular}{||l|rrrrrrrrrrrrr||}
\hline \hline Turn a door knob using my affected hand & 0 & 1 & 2 & 3 & 4 & 5 & 6 & 7 & 8 & 9 & 10 \\
\hline Cut meat using a knife in my affected hand & 0 & 1 & 2 & 3 & 4 & 5 & 6 & 7 & 8 & 9 & 10 \\
\hline Fasten buttons on my shirt & 0 & 1 & 2 & 3 & 4 & 5 & 6 & 7 & 8 & 9 & 10 \\
\hline Use my affected hand to push up from a chair & 0 & 1 & 2 & 3 & 4 & 5 & 6 & 7 & 8 & 9 & 10 \\
\hline Carry a 10lb object in my affected hand & 0 & 1 & 2 & 3 & 4 & 5 & 6 & 7 & 8 & 9 & 10 \\
\hline Use bathroom tissue with my affected hand & 0 & 1 & 2 & 3 & 4 & 5 & 6 & 7 & 8 & 9 & 10 \\
\hline
\end{tabular}

B. USUAL ACTIVITIES

Rate the amount of difficulty you experienced performing your usual activities in each of the areas listed below, over the past week, by circling the number that best describes your difficulty on a scale of 0-10. By "usual activities", we mean the activities you performed before you started having a problem with your wrist. A zero (0) means that you did not experience any difficulty and a ten (10) means it was so difficult you were unable to do any of your usual activities.

\begin{tabular}{||l|rrrrrrrrrrrrr||}
\hline \hline Personal care activities (dressing, washing) & 0 & 1 & 2 & 3 & 4 & 5 & 6 & 7 & 8 & 9 & 10 & \\
\hline Household work (cleaning, maintenance) & 0 & 1 & 2 & 3 & 4 & 5 & 6 & 7 & 8 & 9 & 10 & \\
\hline Work (your job or usual everyday work) & 0 & 1 & 2 & 3 & 4 & 5 & 6 & 7 & 8 & 9 & 10 & \\
\hline Recreational activities & 0 & 1 & 2 & 3 & 4 & 5 & 6 & 7 & 8 & 9 & 10 & \\
\hline
\end{tabular}

(c) JC MacDermid

\section{Figure I}




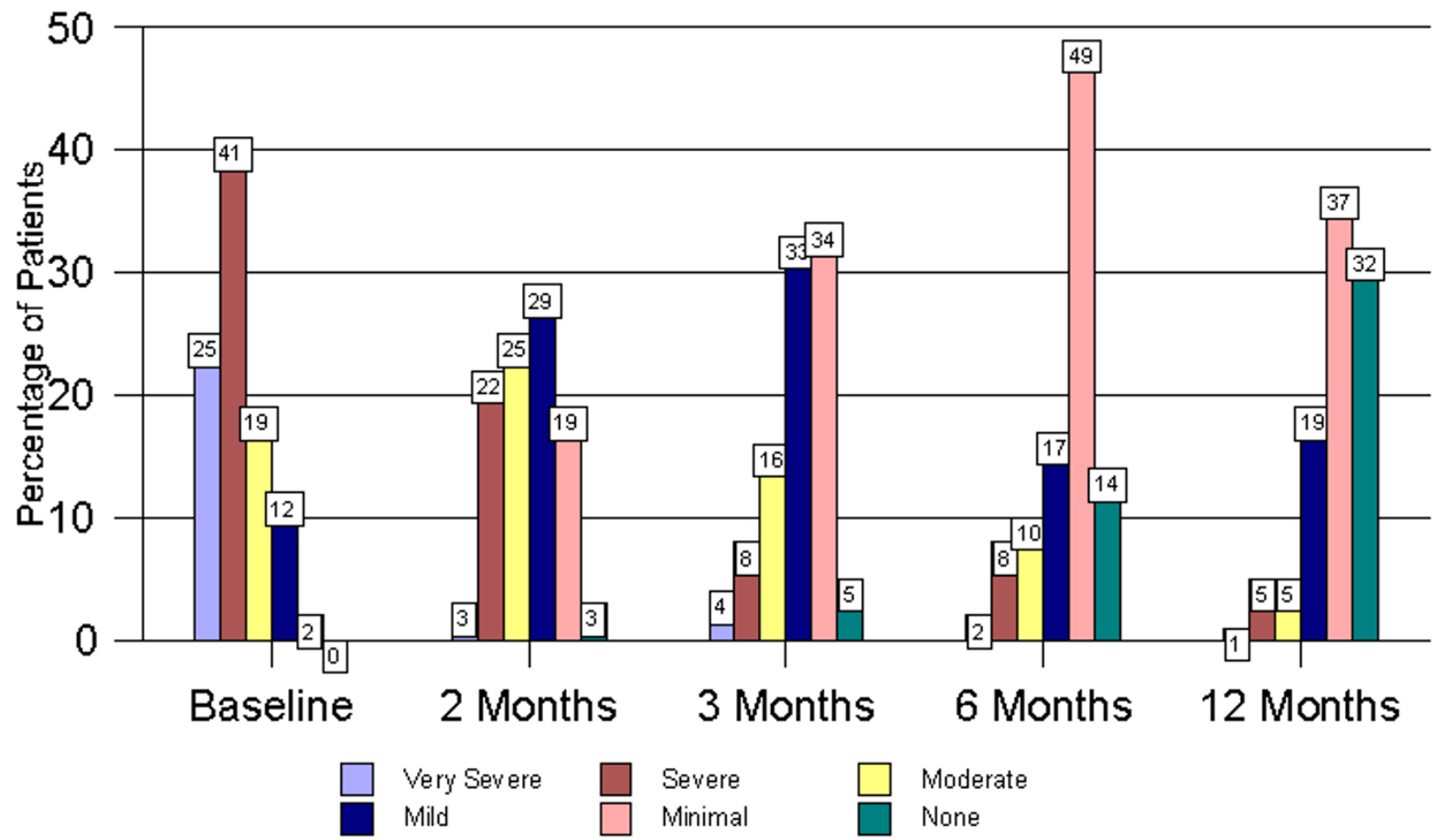

Figure 2

Distribution of Pain Scores Over One-year*

Table I: Descriptors of Severity for Scores for the Patient-rated Wrist Evaluation

\begin{tabular}{llllll}
\hline Descriptor & Item & Pain & Specific Activities & Usual Activities & Total \\
\hline None & 0 & 0 & 0 & 0 & 0 \\
Minimal & $1-2$ & $1-10$ & $0-12$ & $1-8$ & $1-20$ \\
Mild & $3-4$ & $11-20$ & $13-24$ & $9-16$ & $21-40$ \\
Moderate & $5-6$ & $21-30$ & $25-36$ & $17-24$ & $41-60$ \\
Severe & $7-8$ & $31-40$ & $37-48$ & $25-32$ & $61-80$ \\
Very Severe & $9-10$ & $41-50$ & $48-60$ & $32-40$ & $81-100$ \\
\hline
\end{tabular}

Activities subscale where patients rate their difficulty in four domains as compared to their usual level of function/ participation (personal care, household work, work, recreation). Individual subscales can be totaled. A total score (/ 100) for wrist pain and disability is computed by dividing the sum of the 10 functional items by two and adding that subtotal $(/ 50)$ to score for the pain subscale $(/ 50)$. This provides a score from $0-100$, where higher scores indicate greater pain and disability.
Descriptive data analysis of the items on the PRWE was performed using SPSS 11.0. The mean, standard deviation and median scores for each item were calculated at each point in time. The scores of individual items rating pain and disability from $0-10$ were provided qualitative descriptors defined as follows: none (0) minimal (1-2), mild (3-4), moderate $(5-6)$, severe $(7-8)$ or very severe $(9-10)$. For the pain scale item on frequency of pain, the descriptors used were: never (0), rarely (1-2), occasion- 
Table 2: Characteristics of I 29 Patients with Distal Radius Fractures

\begin{tabular}{|c|c|}
\hline Age & 50 years (15); Range $18-78$ \\
\hline Dominance & Right $91 \%$; Left $9 \%$ \\
\hline Sex & Female $68 \%$, Male $32 \%$ \\
\hline Injured Hand & Right $48 \%$, Left $51 \%$, Both I\% \\
\hline Dominant Hand Injured & Yes $51 \%$, No $49 \%$ \\
\hline Worker's Compensated Injury & No $92 \%$, Yes $7 \%$, Pending $1 \%$ \\
\hline Legal Action Involved & No $96 \%$, Yes $1 \%$, Pending $3 \%$ \\
\hline \multirow[t]{3}{*}{ Type of Fracture } & Extra-articular $-23 \%$ \\
\hline & Partial-articular - 39\%, \\
\hline & Complete articular - $38 \%$ \\
\hline \multirow[t]{3}{*}{ Type of Injury } & Low energy (fall from level) $-60 \%$ \\
\hline & Medium (fall from height or with speed) $-33 \%$ \\
\hline & $\begin{array}{l}\text { High (associated with high impact like industrial machine or motor } \\
\text { vehicle accident }-7 \%\end{array}$ \\
\hline \multirow[t]{3}{*}{ Mechanism of Injury } & Fall ice/snow $-21 \%$ \\
\hline & Other fall $-72 \%$ \\
\hline & Other $-7 \%$ \\
\hline \multirow[t]{3}{*}{ Type of Fracture Involved (AO Type) } & A) Extra-articular - $27 \%$ \\
\hline & B) Partial - Articular $36 \%$ \\
\hline & C) Complete articular - $38 \%$ \\
\hline \multirow[t]{8}{*}{ Primary Treatment } & Cast only $-20 \%$ \\
\hline & Reduction plus casting $-31 \%$ \\
\hline & Closed reduction with fixation $-5 \%$ \\
\hline & Arthroscopic reduction and pinning - $9 \%$ \\
\hline & Arthrosporic reduction and external fixation - $15 \%$ \\
\hline & Open reduction and internal fixation $-7 \%$ \\
\hline & Open reduction internal and external fixation $-2 \%$ \\
\hline & Open reduction with bone grating - $11 \%$ \\
\hline \multirow[t]{5}{*}{ Medical problems } & None $-59 \%$ \\
\hline & Heart $-2 \%$ \\
\hline & Arthritis - $9 \%$ \\
\hline & Diabetes - I\% \\
\hline & Other - $29 \%$ \\
\hline Post menopausal (women) & $68 \%$ \\
\hline \multirow[t]{3}{*}{ Occupational demand (hand) } & Low $-42 \%$ \\
\hline & Moderate $29 \%$ \\
\hline & High $-30 \%$ \\
\hline \multirow[t]{8}{*}{ Baseline Work status } & Retired - $25 \%$ \\
\hline & Homemaker - $16 \%$ \\
\hline & Student $-2 \%$ \\
\hline & Unemployed $-4 \%$ \\
\hline & Other medical disability - $3 \%$ \\
\hline & Unable to work due to injury $-33 \% *$ \\
\hline & Working - $18 \%$ \\
\hline & $* 76 \%$ of working patients lost time due to injury \\
\hline \multirow[t]{4}{*}{ Use of Pain medications } & Never $-34 \%$ \\
\hline & Occasionally - $18 \%$ \\
\hline & Daily $-21 \%$ \\
\hline & Several times a day $-26 \%$ \\
\hline Rehabilitation & Formal physiotherapy $-82 \%$ \\
\hline Previous or Current Injury to Same Arm & $20 \%$ \\
\hline Previous or Current Injury to Other Arm & $26 \%$ \\
\hline
\end{tabular}

ally (3-4), frequent (5-6), very frequently (7-8) or constant $(9-10)$ pain. The frequency of these qualitative responses was determined for each time interval. These descriptors were also extended to subscales and the over- all score (See Table 1). The mean scores and the distribution of the categorized scores for subscales and total scores were also calculated. Boxplots of subscale and total scores 
were used to describe the distribution and skew of obtained data.

\section{Results}

Descriptive information on the patients enrolled in this study is described in Table 2. Table 3 contains information on the responses for each individual item of the PRWE scale at each point in time. This indicates, that at baseline, patients had mild pain at rest, frequent pain and that the items "repeated movement/lifting" or "pain at worst" were severe/very severe. A rapid improvement in pain occurred by the two-month time point with patients experiencing occasional pain, minimal pain at rest and moderate pain with exacerbating activities. By 3-months the median response was "no pain at rest" and rarely more intense pain, with pain "at worst" being 3/10.

All specific activities were severely difficult at baseline with the median response being a 10 on all items. A rapid improvement was noted by 2-months and again at the 3month time point. "Pushing up from a chair" and "carrying 10 lbs" remained the most difficult activities throughout recovery. By 12-months after fracture, the median response was zero on all items and a small percentage of patients accounted for the remaining average difficulty (1-2 points) with "carrying $10 \mathrm{lbs}$ " remaining the most difficult task.
Patients' "Usual Role" was moderately affected in personal care, severely affected in ability to perform household duties and very severely affected in work and recreation at baseline. The domains of work and recreation remained the most impaired at each time point. The scores reported by patients reflecting the extent of limitation in their usual role in the 4 domains were lower than their scores for specific activities. Summary scores in subscales and total scores also demonstrated a rapid improvement in pain and disability within the first 3-months following fracture with a positive skew in the data at the six and 12 months time points as indicated by median scores that were lower than the average scores. (Table 5, Figure 7)

Figure 2 demonstrates the distribution of scores on the pain subscale at the five evaluation points over one year. The majority $(66 \%)$ of patients had severe or very severe pain at baseline, whereas pain was moderate or less for $75 \%$ of patients by 2 -months post-fracture. By 6 -months following fracture $63 \%$ of patients had no or minimal pain.

Functional difficulties over time as reported on the PRWE are described in Figures 3 and 4 . At baseline, $86 \%$ of the patients reported very severe disability in the specific activities from the PRWE, while $48 \%$ reported severe disa-

Table 3: Descriptive Information of Item Response on PRWE at Different Time Points

\begin{tabular}{|c|c|c|c|c|c|c|c|c|c|c|c|c|c|c|c|}
\hline \multirow{2}{*}{$\begin{array}{l}\text { Question } \\
\text { Pain Scale }\end{array}$} & \multicolumn{3}{|c|}{ Baseline } & \multicolumn{3}{|c|}{2 Months } & \multicolumn{3}{|c|}{3 Months } & \multicolumn{3}{|c|}{6 Months } & \multicolumn{3}{|c|}{12 Months } \\
\hline & $M n$ & SD & Med & $M n$ & SD & Med & $M n$ & SD & Med & $M n$ & SD & Med & $M n$ & SD & Med \\
\hline At rest & 3.6 & 2.9 & 3 & 1.5 & 2.2 & I & 1.1 & 1.8 & 0 & 0.9 & 1.6 & 0 & 0.7 & 1.5 & 0 \\
\hline Repeat mov"t & 7.8 & 2.5 & 9 & 4.8 & 2.8 & 5 & 3.2 & 2.8 & 4 & 2.7 & 2.7 & 2 & 1.9 & 2.4 & I \\
\hline With lifting & 8.4 & 2.3 & 10 & 5.5 & 3.0 & 5 & 3.9 & 2.9 & 3 & 2.6 & 2.7 & 2 & 1.7 & 2.3 & I \\
\hline At worst & 7.9 & 2.6 & 9 & 5.5 & 2.9 & 5 & 4.0 & 2.9 & 3 & 3.07 & 2.6 & 2 & 2.2 & 2.5 & 2 \\
\hline Frequency & 5.6 & 2.9 & 5 & 3.4 & 2.2 & 3 & 3.0 & 2.5 & 2 & 2.6 & 2.6 & 2 & 1.8 & 2.2 & 1 \\
\hline \multicolumn{16}{|l|}{ Specific Activity } \\
\hline Door knob & 9.2 & 2.1 & 10 & 4.8 & 3.3 & 5 & 2.8 & 2.8 & 2 & 1.8 & 2.4 & I & 1.1 & 2.0 & 0 \\
\hline Cut meat & 9.2 & 2.0 & 10 & 4.7 & 3.5 & 4 & 2.7 & 3.0 & 2 & 1.6 & 2.5 & 0 & 1.2 & 2.2 & 0 \\
\hline Buttons & 8.2 & 2.7 & 10 & 3.2 & 3.0 & 3 & 1.6 & 2.3 & 0 & 1.1 & 2.1 & 0 & 0.8 & 1.7 & 0 \\
\hline Push up -chair & 8.9 & 2.5 & 10 & 5.6 & 3.4 & 5 & 3.2 & 3.0 & 3 & 2.1 & 2.6 & 1 & 1.2 & 2.1 & 0 \\
\hline Carry $10 \mathrm{lbs}$ & 9.5 & 1.7 & 10 & 6.5 & 3.6 & 8 & 4.1 & 3.4 & 3 & 2.6 & 3.1 & I & 1.6 & 2.8 & 0 \\
\hline Bathroom tissue & 9.1 & 2.2 & 10 & 4.7 & 3.9 & 5 & 2.5 & 3.2 & I & 1.6 & 2.6 & 0 & 1.0 & 2.3 & 0 \\
\hline \multicolumn{16}{|l|}{ Usual Activity } \\
\hline Personal care & 6.2 & 2.8 & 6 & 2.7 & 2.9 & 2 & 1.3 & 2.1 & 0 & 0.9 & 1.6 & 0 & 0.6 & 1.3 & 0 \\
\hline Household Work & 7.2 & 2.9 & 8 & 3.7 & 3.1 & 3 & 2.1 & 2.3 & I & 1.4 & 2.1 & 0 & 0.9 & 1.9 & 0 \\
\hline Work & 7.9 & 2.8 & 10 & 4.3 & 3.6 & 4 & 2.5 & 3.0 & I & 1.5 & 2.4 & 0 & 1.0 & 1.8 & 0 \\
\hline Recreation & 7.6 & 3.1 & 9 & 4.3 & 3.4 & 4 & 2.6 & 3.1 & I & 2.0 & 2.8 & I & 1.0 & 2.0 & 0 \\
\hline Pain Score (50) & 33 & 10.8 & 35 & 21.0 & 11.0 & 20 & 15.3 & 11 & 13.5 & 11.7 & 11.2 & 8 & 8.4 & 10.0 & 1 \\
\hline Specific Activity (60) & 54 & 10.8 & 60 & 29.6 & 18.4 & 30 & 16.9 & 15.3 & 13 & 10.8 & 13.4 & 5 & 6.8 & 11.0 & I \\
\hline Usual Activity (40) & 28 & 9.9 & 31 & 15.1 & 11.7 & 14 & 8.6 & 9.5 & 5 & 5.8 & 8.1 & 2 & 3.5 & 6.0 & 0 \\
\hline Total Score (100) & 75 & 17.8 & 79 & 43.3 & 23.0 & 43.5 & 28 & 21.3 & 23 & 20.0 & 20.6 & 12 & 13.5 & 17.0 & I \\
\hline
\end{tabular}

Mn $=$ Mean, Med $=$ Median, $S d=$ standard deviation 


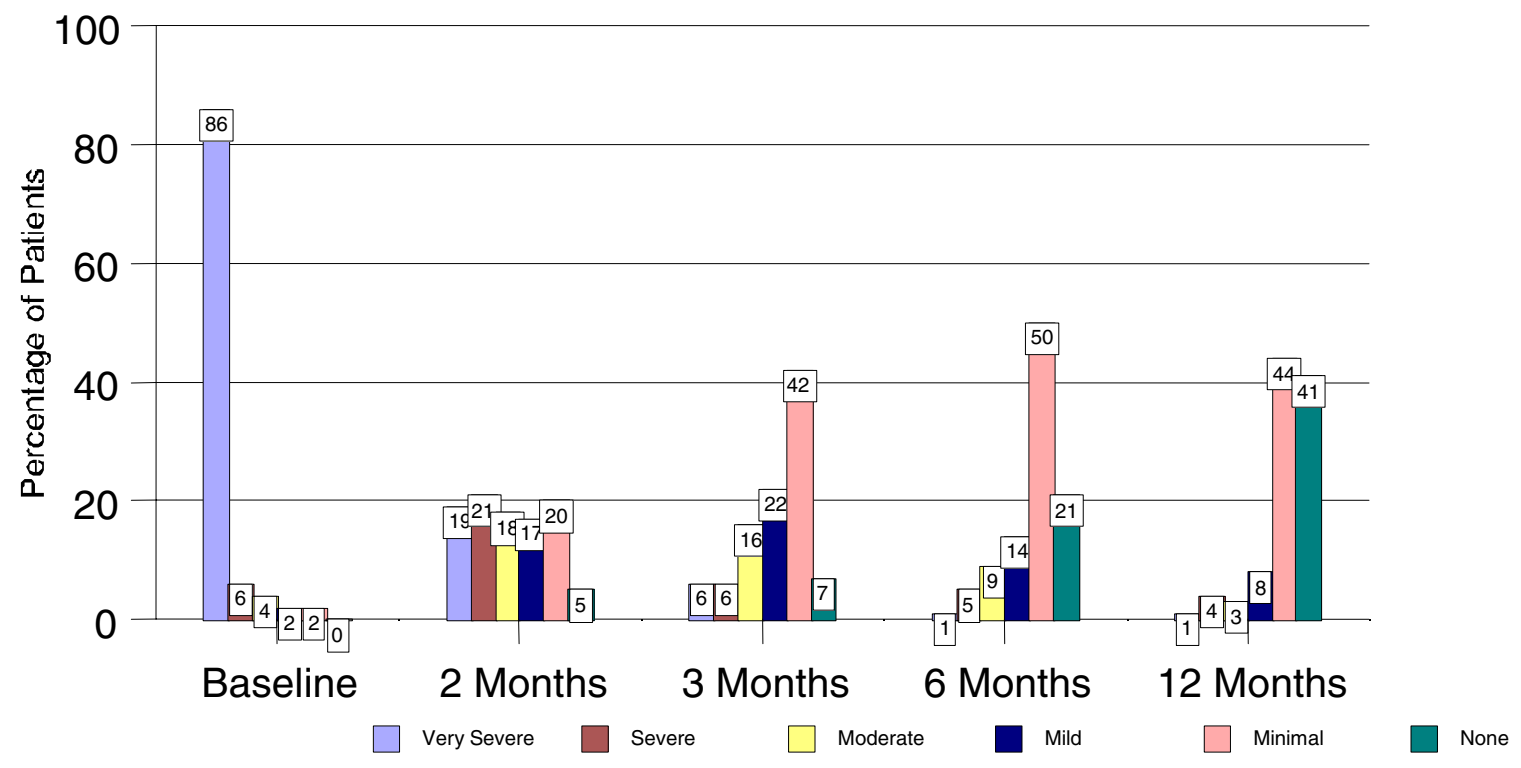

Figure 3

Distribution of Difficulty with Specific Activities over One-year*

bility performing their usual activities in the domains of personal, housework, work and recreation. The inability to perform usual activities (participation) was lower at each successive time points. By 3 -months $49 \%$ of the patients had minimal or no difficulty performing these 6 specific activities and $64 \%$ reported minimal/no difficulty with their usual activity. One-year following fracture, disability was mild. However, only $41 \%$ of patients reported no difficulty with specific activities and only 54\% reported no difficulty with their usual activities.

The total pain and disability scores summarize these trends. At baseline, $81 \%$ of patients experienced severe or very severe pain and disability, which improved dramatically in the first 3-months, followed by slower improvements such that at 1 year following a fracture, $79 \%$ of patients have no/minimal pain and disability. However, $3 \%$ of the patients continued to have moderate, $4 \%$ severe and $1 \%$ very severe pain and disability even at this late stage. These trends are evident by the skew in PRWE scores where outliers at 1-year are those with high scores, whereas outliers at baseline tended to include low scores. (Figures 6 and 7)

\section{Discussion}

This study provided information on the pain and disability experience of patients following a distal radius fracture and how it changes over time. The data demonstrate that patients presenting at a baseline clinic visit, following reduction of their fracture, exhibit high levels of pain and disability and have very severe difficulty performing the 6 specific tasks listed on the PRWE. High levels of pain and disability coincide with the reparative phase where, soft tissue and bone healing would be occurring. While the self-report measure quantified the intensity of the pain experienced, it could not identify the location or source of 


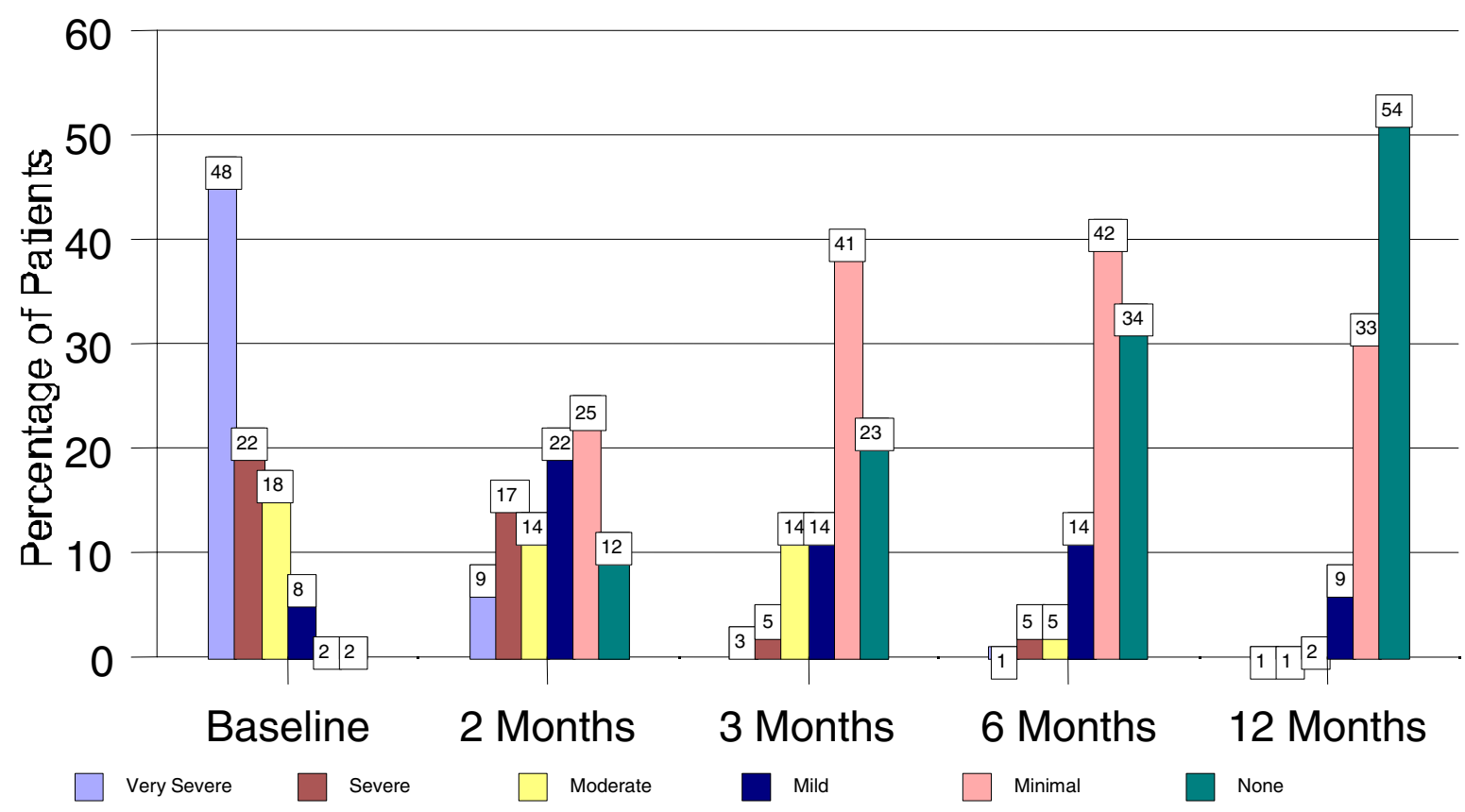

Figure 4

Distribution of Difficulty with Usual Activities over One-year*

this pain, so pain from both bone and soft tissue are implicated. Following tissue healing, "a rehabilitative phase" is characterized by slower, continued improvement in pain and disability with the majority of recovery occurring by 6 months post fracture. A small minority of patients continue to have significant pain and disability at one year following their injury. Carrying weight is the most difficult task for patients, whereas, work and recreation are the domains of life that are most severely and continuously affected during their recovery. Previous studies investigating the relationship between impairment and disability have suggested that strength is correlated to functional ability, which is consistent with these findings. $[1,2]$

Functional difficulties reported on the specific activities subscale of the PRWE presented very severe difficulty at baseline. In fact, the median response was 10 (unable to do) for all specific items at baseline. This is logical given that items like repeated movement or lifting would be contra-indicated while the fracture was still healing. Furthermore, the majority of patients would be immobilized in casts, external fixators and/or through internal hardware which would limit the ability to move. The fact that the six items from the Specific Activities subscale presented a greater amount of difficulty to patients than their general level of disability in the domains of personal, household work, work, and recreation reported on the Usual Activities Subscale, supports the validity of the PRWE by indicating that the item reduction process correctly identified activities which are particularly difficult for patients with wrist pathology.

Two months later, with removal of these devices, a large improvement in function should be anticipated. At this time, carrying a ten-pound object, pushing up from a 


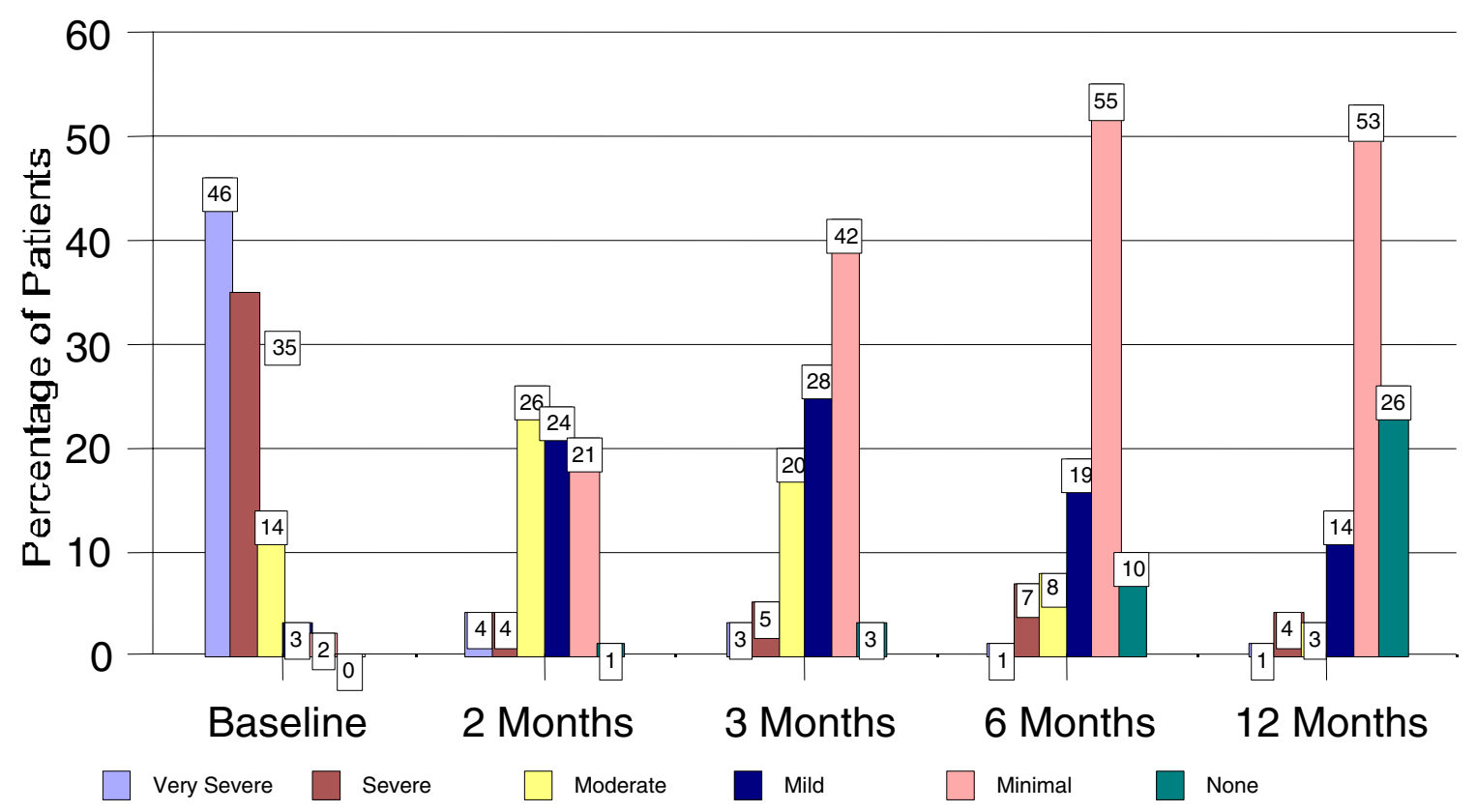

Figure 5

Distribution of PRWE Scores over One-year*

chair, turning a doorknob and bathroom hygiene still presented moderate difficulty for patients. The least disability was reported in fastening buttons. This item is considered to reflect difficulty in fine dexterity which might only be notably compromised in complicated cases, e.g. with median nerve problems, associated nerve injury or reflex sympathetic dystrophy. As these complications are rare [12], it is not surprising that this item would have lowest mean/frequency of reported difficulty.

The amount of restriction from usual activity reported at baseline in 4 domains of daily life was less than reported for specific tasks. This likely reflects the fact that patients can use their injured hand, to a certain extent, with their uninjured hand to perform tasks of daily life. The fact that level of disability reported in the items on the Usual Activity subscale is lower, at each time point, than for the items on the Specific Activities subscale also supports the concept that the impact of specific impairments varies according to the individual's normal role and environment $[13,14]$. It is of particular importance when evaluating individual patients to be able to examine both the specific activities that are difficult for a patient, as well as their overall difficulty in completing their normal activities and roles. This data suggests that patients can distinguish their capability in specific activities from their overall participation in domains of life.

The results of this study are in agreement with qualitative work reported by Bialocerkowski [8], on a group of mixed wrist disorders. As in the current study, these authors reported that patients found work activities and household work (domestic duties) to present greater difficulty than personal care activities. During their open-ended qualitative interviews, patients recorded difficulty with activities similar to those represented on the PRWE. For 


\section{Baseline Subscale and Total PRWE Scores}

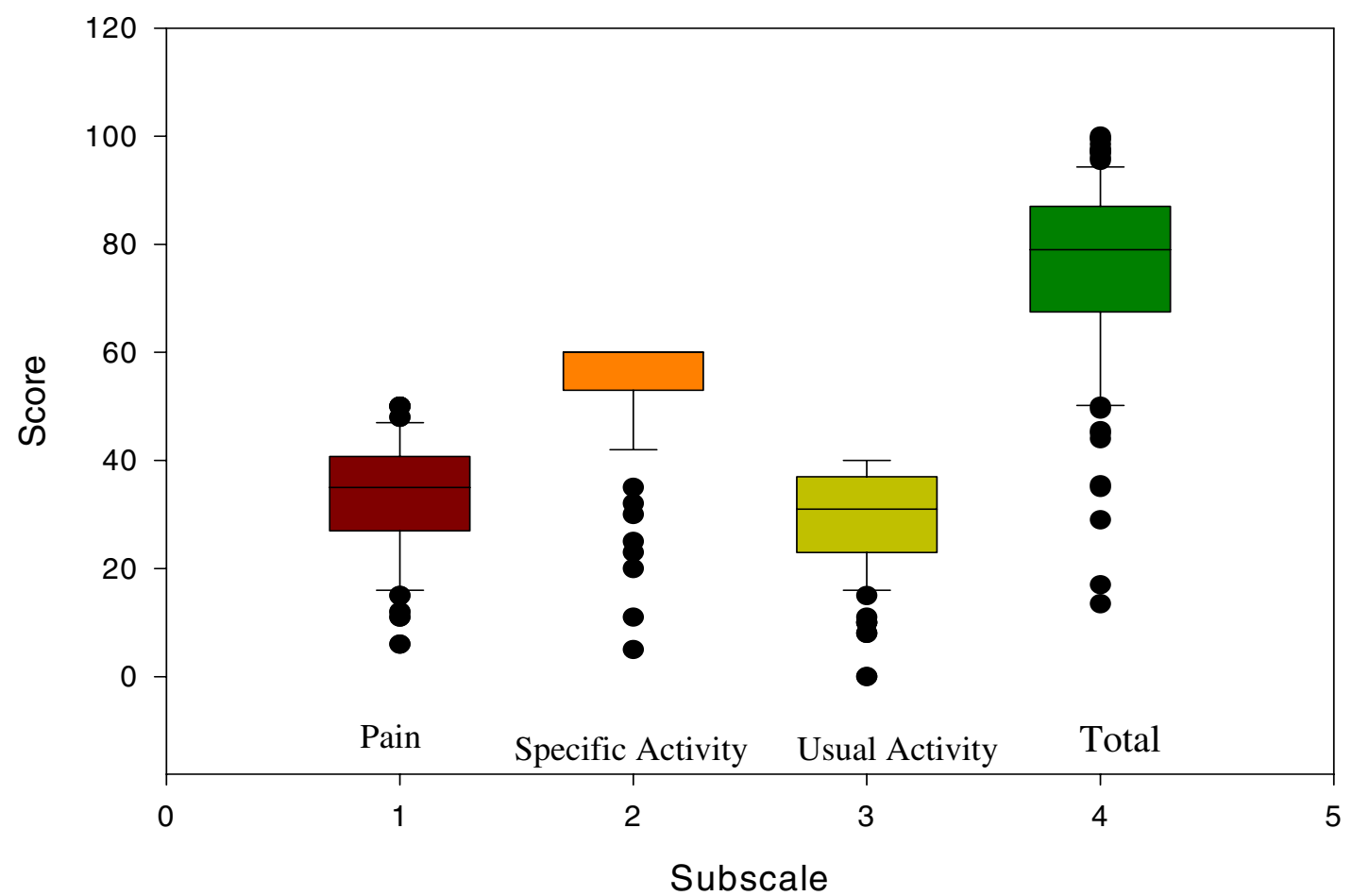

Figure 6

Graph of Baseline Subscale and Total PRWE Scores*

example cutting meat with a knife (using eating utensils), turning a doorknob (open/close doors), buttons (handle buttons), push up from a chair (not mentioned), carry ten pounds (carry a child/saucepan/work activities involving grasping and lifting) and bathroom hygiene (washing the body) appear on both the PRWE and the data reported in the interviews. The similarity between items elicited during semi-structured interviews conducted by independent authors and those represented on the PRWE supports the validity of the questionnaire in measuring the wristrelated disability.

While Bialocerkowski [8] found similar functional disabilities in patients with wrist disorders as those included on the PRWE, her study was cross-sectional and thus did not elucidate how these particular disabilities changed over time. The current study provides additional information on the rate of improvement of both pain and disabil- ity during the year following a distal radius fracture. This information is more specific to distal radius fractures than previous work utilizing a study sample consisting of a variety of wrist problems. The qualitative work conducted by Bialocerkowski [8] used selective sampling and excluded patients if they had any systemic or neurological conditions, any wrist problems on the other hand or any other hand or arm problems. This type of selective sampling strategy is common in qualitative studies and entirely appropriate to that methodology. However, the results can only be generalized to the population studied. In contrast, this cohort study incorporated all patients who were able to complete the questionnaire, either independently or through the use of an interpreter and thus can be generalized to the broad spectrum of patients with distal radius fractures seen in a hand clinic. From this study, we are able to conclude that the majority of patients, should experience the majority of their improve- 


\section{1-Year Subscale and PRWE Scores}

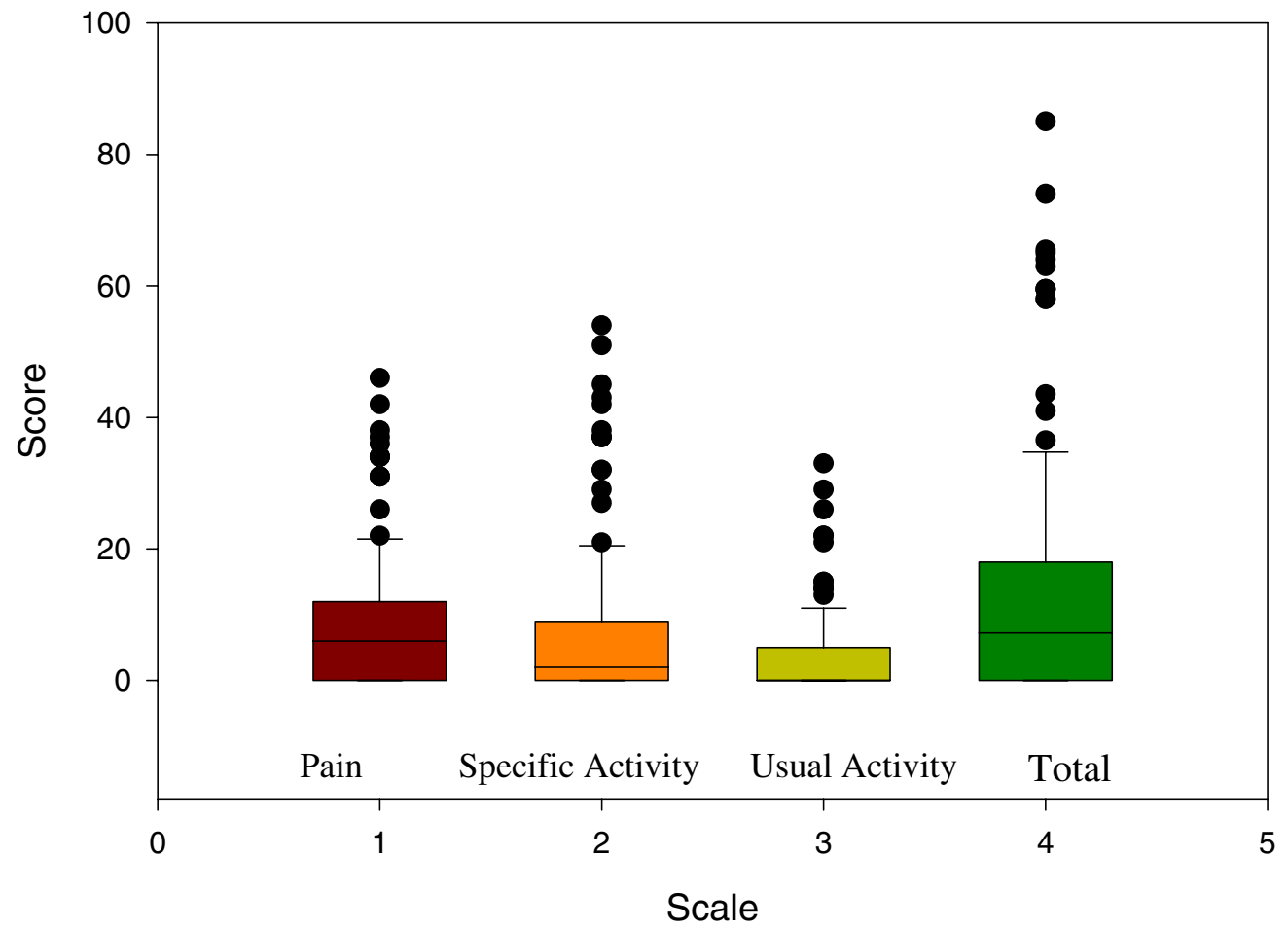

Figure 7

Graph of I-year Subscale and Total PRWE Scores*

ment within six-months post-fracture, but a minority of patients will have lasting difficulties that persist for one year. While this group was too small within this study to determine specific reasons for poorer outcome, our previous work has shown that secondary compensation, more severe fracture displacement and patient education level are determinants of higher reported pain and disability. [2]

Bialocerkowski [8] also demonstrated that patients use a variety of compensatory mechanisms such as using the other hand, taking longer to do some tasks or doing things in a different way as common strategies when dealing with their wrist impairments. This provides an explanation for why different patients report different levels of disability even when impairments are very similar. They were also able to demonstrate that nonphysical, mostly negative, effects can occur following a wrist fracture. The most frequent of these was a financial burden (5/43). Aspects of how patients deal with their disabilities were not addressed within the current study.

Despite the fact that their qualitative interviews support the content of the PRWE, Bialocerkowski [8] suggested that currently available standardized instruments are deficient because "no published wrist or hand instrument covers the full extent of the activities, as identified by our sample." While qualitative research describing the individual disability experience is highly useful and brings a richer understanding of the problems experienced by patients, this does not diminish the importance of validated self-report scales as a means to describe disability or to report outcomes. Sampling an appropriate number of disabilities which are known to be difficult for most patients with wrist disorders provides a means of describing pain and disability in a standardized way that is repre- 
sentative, although not fully comprehensive. It is noteworthy that patient-specific measures have been developed [15] which allow one to customize items according to the individual patient's priorities. Future studies which aim to describe the disability experience of upper extremity disorders, in more detail, could consider using both the PRWE and the Patient-Specific Scale.

The data reported in this study are useful to understand the disability caused by a distal radius fracture. Additionally, this data could be used in clinical decision-making. Comparing scores of individual patients to the median score reported by patients in this study (matched according to a time interval) will help identify whether that individual patient is reporting an atypical disability experience. For example, if a clinician observes that an individual patient is reporting abnormally high scores for pain at rest at a baseline visit, they may become concerned that this patient is presenting with early signs of complex regional pain syndrome and look for other signs/evidence suggestive of this problem. They might alter the treatment plan in light of these findings. Similarly, patients who do not exhibit substantial improvement in their scores between their baseline and 2-month evaluations may also generate concern. More intensive therapy may be indicated, further investigation to look for undetected associated injuries or complications might be appropriate; or the clinician may need to investigate the role of nonmedical issues that could be contributing to the patient's disability. Conversely, patients with pain and disability scores that are exceptionally low may not require in-house therapy, may return to work earlier or be followed less closely. Studies which investigate the appropriate cut-off points and accuracy of outcome scales when used in these ways would provide evidence upon which to base clinical-decision making.

Ideally self-report scales should help us understand the disability experienced by the individual patient, document the effectiveness of their treatment and assist with clinical-decision making during recovery. While there is an increased utilization of self-report scales to document the effectiveness of interventions, relatively little research has focused on how these instruments can help us to understand the patient's disability experience or contribute to decision-making during their care. While studies which report minimally important clinical differences or measurement error are essential to help clinicians evaluate significant clinical change following interventions, clinicians must also understand the normal course of recovery following an injury so that they can judge when a given patient deviates from these expectations. Future investigations that enhance our ability to understand the disability caused by specific musculoskeletal disorders and the impact of self-report scales on clinical decision- making/patient outcomes could contribute to providing more effective patient-centred care.

\section{Conclusions}

Patients experience profound pain and disability immediately following a distal radius fracture. The normal course of recovery is for symptoms to become mild within three months. Prolonged pain or disablement occurs for a minority of patients. Clinicians can compare their patient responses to the data provided in this study to identify patients reporting atypical experiences. Atypical responses may indicate the need for further evaluations, changes in treatment programs or modification of plans for return to work.

\section{Competing interests}

None of the authors have received any reimbursement for this study that would be affected by its publication. None hold any stock or shares in any organization that would gain or lose from publication of this paper. There are no financial competing interests. There are no non-financial competing interests.

\section{Author's Contributions}

Joy MacDermid developed the instrument that was used to collect data, designed the study protocol, supervised its execution, analyzed the data, wrote the first draft for publication and made revisions in response to reviewers.

James Roth enrolled, treated and evaluated patients in this study. He contributed to the design of evaluation procedures, study equipment and personnel funding and revisions to both the initial and revised draft manuscript.

Robert Richards enrolled, treated and evaluated patients in this study. He contributed to the design of evaluation procedures, study equipment and personnel funding and revisions of the both the initial and revised manuscripts.

\section{Acknowledgments}

The authors would like to thank Jan Smith for her contribution to the study which involved patient testing and data entry. We thank the American Society for Surgery of the Hand (Outcomes Grant) for supporting this work. We thank Drs Faber, King, Gan, Patterson and Ross for enrolling patients in this study.

\section{References}

I. Karnezis IA and Fragkiadakis EG: Association between objective clinical variables and patient-rated disability of the wrist. J Bone Joint Surg Br 2002, 84:967-970.

2. MacDermid JC, Donner A, Richards RS and Roth JH: Patient versus injury factors as predictors of pain and disability six months after a distal radius fracture. J Clin Epidemiol 2002, 55:849-854.

3. MacDermid JC, Richards RS and Roth JH: Distal radius fracture: a prospective outcome study of $\mathbf{2 7 5}$ patients. J Hand Ther 200I, 14:154-169.

4. Hildebrand KA, Ross DC, Patterson SD, Roth JH, MacDermid JC and King G]: Dorsal perilunate dislocations and fracture-disloca- 
tions: questionnaire, clinical, and radiographic evaluation. J Hand Surg [Am ] 2000, 25:1069-1079.

5. Konrath GA and Bahler $\mathrm{S}$ : Open reduction and internal fixation of unstable distal radius fractures: results using the trimed fixation system. J Orthop Trauma 2002, 16:578-585.

6. Jupiter JB, Ring D and Weitzel PP: Surgical treatment of redisplaced fractures of the distal radius in patients older than $\mathbf{6 0}$ years. J Hand Surg [Am ] 2002, 27:7|4-723.

7. MacDermid JC, Richards RS, Donner A, Bellamy $\mathrm{N}$ and Roth $\mathrm{JH}$ : Responsiveness of the short form-36, disability of the arm, shoulder, and hand questionnaire, patient-rated wrist evaluation, and physical impairment measurements in evaluating recovery after a distal radius fracture. J Hand Surg [Am ] 2000, 25A:330-340.

8. Bialocerkowski AE: Difficulties associated with wrist disorders - a qualitative study. Clin Rehabil 2002, 16:429-440.

9. MacDermid JC, Turgeon T, Richards RS, Beadle $M$ and Roth JH: Patient rating of wrist pain and disability: a reliable and valid measurement tool. J Orthop Trauma 1998, I 2:577-586.

10. MacDermid JC: Development of a scale for patient rating of wrist pain and disability. J Hand Ther 1996, 9: 178-183.

11. MacDermid JC and Tottingham V: Responsiveness of the DASH and PRWE in Wrist or Hand Injured Patients Referred for Rehabilitation. J Hand Ther 2004 in press.

12. McKay SD, MacDermid JC, Roth JH and Richards RS: Assessment of complications of distal radius fractures and development of a complication checklist. J Hand Surg [Am] 200I, 26:916-922.

13. Jette AM: Physical disablement concepts for physical therapy research and practice ]. Phys Ther 1994, 74:380-386.

14. Heekens YF, Brandsma JW, Lakerveld-Heyl K and Ravensberg CD: Impairments and disabilities - The difference: Proposal for adjustment of the international classification of impairments, disabilities and handicaps. Phys Ther 1994, 74:430-442.

15. Stratford P, Gill C, Westway M and Binkley J: Assessing disability and change of individual patients: $A$ report of a patient specific measure. Physiother Can 1995, 47:258-263.

\section{Pre-publication history}

The pre-publication history for this paper can be accessed here:

http://www.biomedcentral.com/1471-2474/4/24/prepub

\section{Publish with Bio Med Central and every scientist can read your work free of charge}

"BioMed Central will be the most significant development for disseminating the results of biomedical research in our lifetime. "

Sir Paul Nurse, Cancer Research UK

Your research papers will be:

- available free of charge to the entire biomedical community

- peer reviewed and published immediately upon acceptance

- cited in PubMed and archived on PubMed Central

- yours - you keep the copyright 